INPLASY

PROTOCOL

To cite: Lu et al. Safety and efficacy of Auricular acupuncture in patients with depression after percutaneous coronary intervention:A protocol for systematic review and meta-analysis. Inplasy protocol 202230003. doi: 10.37766/inplasy2022.3.0003

Received: 01 March 2022

Published: 01 March 2022

Corresponding author:

Lu Ruozhu

532364820@qq.com

Author Affiliation: Changchun University of Chinese Medicine.

Support: NSFC (No.82174350).

Review Stage at time of this submission: Preliminary searches.

Conflicts of interest: None declared.

\section{Safety and efficacy of Auricular acupuncture in patients with depression after percutaneous coronary intervention: A protocol for systematic review and meta-analysis}

Lu, R'; Shi, R²; Zhang, M³; Shao, X4; Xue, W5; Guo, Q6; Wang, C7; Deng, $Y^{8}$.

Review question / Objective: P:depression in post-PCI patients; I: auricular acupuncture combining or not combining with western medicines; C:Conventional western medicine treatment as the sole treatment.; O:The primary outcomes are the change in Hamilton Depression Rating scale (HAMD) score and the Self-Rating Depression Scale (SDS) score.The secondary outcomes of this review mainly include the following aspects:1. All-cause mortality, cardiac mortality. 2. Major Adverse Cardiovascular Events (MACEs). 3. Rehospitalization rate. 4. Quality of Life Scale (SF-36). Effective rate, adverse event rate; S: All relevant randomized controlled trials (RCTs) in English and Chinese will be included. While NonRCTs, quasi-RCTs, cohort studies, reviews, case reports, experimental studies, expert experience, the data of the included study is missing or incomplete, and duplicate publications will be excluded to ensure the quality of this systematic review.

INPLASY registration number: This protocol was registered with the International Platform of Registered Systematic Review and Meta-Analysis Protocols (INPLASY) on 01 March 2022 and was last updated on 01 March 2022 (registration number INPLASY202230003).

\section{INTRODUCTION}

Review question / Objective: Percutaneous coronary intervention $(\mathrm{PCl})$ has been increasingly applied as an effective revascularization strategy in patients with coronary artery disease (CAD). However, recent studies had indicated a higher incidence of depression on post-PCI patients. Acupuncture therapy is effective for depression. As a form of acupuncture, the auricular acupuncture has been used to relieve symptoms in patients with post-PCI depression, but its effectiveness and safety 
have not yet reached a definitive conclusion.Therefore, this systematic review and meta-analysis protocol is planned to evaluate the efficacy and safety of auricular acupuncture for depression in post-PCI patients.Percutaneous coronary intervention ( $\mathrm{PCl}$ ) has been increasingly applied as an effective revascularization strategy in patients with coronary artery disease (CAD). However, recent studies had indicated a higher incidence of depression on post-PCI patients. auricular acupuncture therapy is effective for depression. However, the treatment effect of depression on post-PCI patients is still not clear. Therefore, this systematic review and meta-analysis protocol is planned to evaluate the efficacy and safety of auricular acupuncture for depression in post-PCI patients. Objective: P:depression in postPCI patients; I: auricular acupuncture combining or not combining with western medicines; C:Conventional western medicine treatment as the sole treatment.; $0:$ The primary outcomes are the change in Hamilton Depression Rating scale (HAMD) score and the Self-Rating Depression Scale (SDS) score.The secondary outcomes of this review mainly include the following aspects:1. All-cause mortality, cardiac mortality. 2. Major Adverse Cardiovascular Events (MACEs). 3. Rehospitalization rate. 4. Quality of Life Scale (SF-36). Effective rate, adverse event rate; $S$ : All relevant randomized controlled trials (RCTs) in English and Chinese will be included. While NonRCTs, quasi-RCTs, cohort studies, reviews, case reports, experimental studies, expert experience, the data of the included study is missing or incomplete, and duplicate publications will be excluded to ensure the quality of this systematic review.

Rationale: With the advantage of miniature damage and optimal effectiveness, percutaneous coronary intervention (PCI) has been performed in a high number of coronary artery disease (CAD) patients.Many studies have demonstrated increased prevalence of depressive disorder in patients with $\mathrm{Ml}$ or received $\mathrm{PCl}$ as well as poor outcomes in the long-
term.Antidepressants have potential adverse effects such as sexual dysfunction, weight changes and sleep disruption.Acupuncture therapy is effective for depression. As a form of acupuncture, the auricular acupuncture has been used to relieve symptoms in patients with post-PCI depression, but its effectiveness and safety have not yet reached a definitive conclusion.Therefore, this systematic review and meta-analysis protocol is planned to evaluate the efficacy and safety of auricular acupuncture for depression in post-PCI patients.

Condition being studied: Percutaneous coronary intervention (PCI) has been increasingly applied as an effective revascularization strategy in patients with coronary artery disease (CAD). However, recent studies had indicated a higher incidence of depression on post-PCI patients. At present, the drug treatments of post-PCI depression are difficult to achieve satisfactory results, and the side effects are obvious. Acupuncture therapy is effective for depression. As a form of acupuncture, the auricular acupuncture has been used to relieve symptoms in patients with post- $\mathrm{PCl}$ depression, but its effectiveness and safety have not yet reached a definitive conclusion.Therefore, this systematic review and meta-analysis protocol is planned to evaluate the efficacy and safety of auricular acupuncture for depression in post- $\mathrm{PCl}$ patients.

\section{METHODS}

Participant or population: Depression in post-PCI patients.

Intervention: Auricular acupuncture combining or not combining with western medicines.

Comparator: Conventional western medicine treatment as the sole treatment.

Study designs to be included: All relevant randomized controlled trials (RCTs) in English and Chinese will be included. While NonRCTs, quasi-RCTs, cohort studies, reviews, case reports, experimental 
studies, expert experience, the data of the included study is missing or incomplete, and duplicate publications will be excluded to ensure the quality of this systematic review.

Eligibility criteria: All relevant randomized controlled trials (RCTs) in English and Chinese will be included. While NonRCTs, quasi-RCTs, cohort studies, reviews, case reports, experimental studies, expert experience, the data of the included study is missing or incomplete, and duplicate publications will be excluded to ensure the quality of this systematic review.

Information sources: Six English databases (PubMed, Web of science, Medline, EMBASE, Springer Cochrane Library, and WHO International Clinical Trials Registry Platform) and 4 Chinese databases (Wan fang Database, Chinese Scientific Journal Database, CNKI, and Chinese Biomedical Literature Database) will be searched normatively in accordance with the rule of each database from the inception to February 1, 2022.

Main outcome(s): The primary outcomes are the change in Hamilton Depression Rating scale (HAMD) score and the SelfRating Depression Scale (SDS) score.

Additional outcome(s): The secondary outcomes of this review mainly include the following aspects:1. All-cause mortality, cardiac mortality. 2. Major Adverse Cardiovascular Events (MACEs). 3. Rehospitalization rate. 4. Quality of Life Scale (SF-36).

Data management: Relevant literatures will be obtained from specified databases, later imported into an Endnote X9 created database. Duplicate documents will be screened out through this process. Independent screening of titles, abstracts, and keywords of all retrieved records will be performed by 2 researchers. The name of the study, author, publishing year, country, database, and justification of the study meeting eligibility criteria to be therefore included in the review will be documented within an excel spreadsheet.
Reasons of inclusion and exclusion (PICOS) are disclosed in a spreadsheet during abstract screening and full-text evaluation. A third researcher will be required on making the final decision to resolve any disagreement among the 2 researchers on literatures.

Quality assessment / Risk of bias analysis: The basic process of including literature will be pursued in reference to the Cochrane Collaboration System Evaluator's Manual (5.1.0).

Strategy of data synthesis: Data analysis and synthesis will be performed using RevMan version 5.3 software provided by the Cochrane Collaboration. The software will be used to obtain forest plots and test the heterogeneity between the included studies. Risk ratio (RR) with $95 \%$ Cls will be used for dichotomous data, while the continuous data will be analyzed by mean difference (MD) or standard MD (SMD) with 95\% Cls. Heterogeneity will be assessed by visual inspection of the forest plots and detected by standard $X^{2}$ test and 12 test. When $P>0.1,12<50 \%$, it will be considered as no significant heterogeneity between the trials, and the fixed effect model will be applied for statistics, otherwise, the random effect model will be chosen. When heterogeneity occurs, sensitivity analysis or meta regression will be performed to assess the source of heterogeneity.

Subgroup analysis: When heterogeneity is detected, subgroup analysis will be used (e.g., different types of western medicines therapies, patient conditions, research quality, publication age, and participation population) to spot the source of heterogeneity.

Sensitivity analysis: In trials with sufficient data,sensitivity analyses will be taken to test the robustness and reliability of the results. Our sensitivity analysis will be based on heterogeneity, sensitivity analysis may be performed, and certain low-quality or unblinded studies would be excluded when heterogeneity occurs.

Country(ies) involved: China. 
Keywords: auricular acupuncture, depression, percutaneous coronary intervention, protocol, systematic review.

Contributions of each author:

Author 1 - Lu Ruozhu.

Email: 532364820@qq.com

Author 2 - Shi Rui.

Email: 4352637@qq.com

Author 3 - Zhang Miao.

Email: zmiao0606@163.com

Author 4 - Shao Xiao.

Email: 859585592@qq.com

Author 5 - Que Wen.

Email: 1454650140@qq.com

Author 6 - Guo Qian.

Email: 1051248945@qq.com

Author 7 - Wang Cheng.

Email: 519081091@qq.com

Author 8 - Deng Yue.

Email: dyue7138@sina.com 